Chirurg 2019 90 (Suppl 2):S40 https://doi.org/10.1007/s00104-019-0889-1 Online publiziert: 13. Februar 2019

(c) Springer Medizin Verlag GmbH, ein Teil von Springer Nature 2019

\section{Originalpublikation}

Jayne D, Pigazzi A, Marshall $\mathrm{H}$ et al (2017) Effect of robotic-assisted vs conventional laparoscopic surgery on risk of conversion to open laparotomy among patients undergoing resection for rectal cancer. JAMA 318:1569. https://doi.org/10.1001/jama.2017.7219

Hintergrund. In den vergangenen Jahren hat die roboterassistierte Rektumresektion (RAR) an Popularität gewonnen. Aufgrund der dreidimensionalen Sicht und einem größeren Bewegungsspektrum der Instrumente besitzt die RAR das Potenzial, einige Einschränkungen der konventionellen laparoskopischen Rektumresektion (LR) zu überwinden. Bisher gibt es jedoch wenig Daten, die einen Vorteil der RAR belegen.

Methoden. Die ROLARR-Studie (Robotic vs. Laparoscopic Resection for Rectal Cancer) ist eine multizentrische randomisierte Studie zum Vergleich RAR und LR. Eingeschlossen wurden Patienten mit einem Adenokarzinom des Rektums ( $\leq 15 \mathrm{~cm}$ von der Anokutanlinie) und kurativer Resektions- oder Exstirpationsmöglichkeit. Primärer Endpunkt war die Konversionsrate zur offenen Operation.

Ergebnisse. Insgesamt wurden $471 \mathrm{~Pa}$ tienten in die Studie eingeschlossen. Die Konversionsrate betrug 10,1\% und

Dieser Beitrag wurde erstpubliziert in Der Chirurg (2018) 89:390-390. https://doi.org/10. 1007/s00104-018-0642-1

M. Schrempf $\cdot$ M. Anthuber

Klinik für Allgemein-, Viszeral- Transplantationschirurgie, Klinikum Augsburg, Augsburg, Deutschland

\title{
Roboterassistierte vs. laparoskopische Rektumresektion - Einfluss auf die Konversionsrate
}

unterschied sich nicht zwischen der RAR-Gruppe (12,2\%) und der LRGruppe (8,1\%; adjustiertes Odds Ratio $[\mathrm{aOR}]=0,61 ; \quad 95 \%$-Konfidenzintervall [KI] $0,31-1,21 ; p=0,16)$. Auch hinsichtlich der intraoperativen und postoperativen Komplikationsrate, der Rate an positivem zirkumferenziellem Resektionsrand (CRM), der 30-Tage-Letalität sowie der Sexual- und Blasenfunktion war kein Unterschied zwischen den beiden Gruppen nachweisbar. Die durchschnittliche Operationszeit war in der RAR-Gruppe 37,5 min länger als in der LR-Gruppe (298,5 min vs. $261 \mathrm{~min})$. Unabhängige Risikofaktoren für eine Konversion zur offenen Operation waren männliches Geschlecht (aOR=2,44; $95 \%$-KI 1,05-5,71; $p=0,04)$ sowie ein $\mathrm{BMI}>30(\mathrm{aOR}=4,69$; $95 \%$-KI 2,08-10,58; $p<0,001)$. Ebenso war die Konversionsrate bei Patienten mit Rektumresektion im Vergleich zu Patienten mit Rektumexstirpation höher $(\mathrm{aOR}=5,44 ; 95 \%$-KI $1,60-18,52$; $p=0,007)$.

Diskussion und Fazit. In dieser Arbeit wurde hinsichtlich der Konversionsrate kein Vorteil der RAR im Vergleich zur LR festgestellt. Die Kosten der RAR im Vergleich zur LR waren pro Fall um $£ 980$ bzw. \$ 1132 höher. Obwohl im Rekrutierungszeitraum durchschnittlich weniger als 5 Patienten pro Zentrum eingeschlossen wurden, war die Rate an positiven CRM mit 5,7\% deutlich niedriger als in den Laparoskopie-Armen der beiden multizentrischen Studien ACOSOGZ6051 (12,1\%) und ALaCaRT (7\%), die einen Vorteil der konventionellen la- paroskopischen Resektion im Vergleich zur offenen Rektumresektion infrage stellen $[1,2]$. Zu beachten ist, dass die teilnehmenden Chirurgen im Median 45\% weniger roboterassistierte Fälle operiert hatten und somit mehr Erfahrung in der konventionellen Laparoskopie besaßen. Die unterschiedliche Erfahrung der Chirurgen mit den beiden Methoden könnte $\mathrm{zu}$ einem Bias in der Studie beitragen. Obwohl die Kurzzeitergebnisse der ROLARR-Studie aktuell gegen einen Vorteil der RAR sprechen, bietet die roboterassistierte Chirurgie ein Potenzial für die Zukunft. Um dieses Potenzial voll ausschöpfen zu können, ist allerdings eine strukturierte und frühe Ausbildung von Chirurgen in der roboterassistierten Chirurgie notwendig [3].

\section{Korrespondenzadresse}

Prof. Dr. M. Anthuber

Klinik für Allgemein-, Viszeral- Transplantationschirurgie, Klinikum Augsburg Stenglinstr. 2, 86156 Augsburg, Deutschland matthias.anthuber@klinikum-augsburg.de

Interessenkonflikt. M. Schrempf und M. Anthuber geben an, dass kein Interessenkonflikt besteht.

\section{Literatur}

1. Fleshman J, Branda M, Sargent DJ et al (2015) Effect of laparoscopic-assisted resection vs open resection of stagell or III rectal cancer on pathologic outcomes. JAMA 314:1346

2. Stevenson ARL, Solomon MJ, Lumley JW et al (2015) Effect of laparoscopic-assisted resection vs open resection on pathological outcomes in rectal cancer. JAMA 314:1356

3. Vetter MH, Green I, Martino M et al (2015) Incorporating resident/fellow training into a robotic surgery program. J Surg Oncol 112:684-689 